\title{
Association of Kidney Dysfunction With Asymptomatic Cerebrovascular Abnormalities in a Japanese Population With Health Checkups
}

\author{
Kaori Hayashi, MD, PhD; Michiyo Takayama, MD, PhD; Takeshi Kanda, MD, PhD; \\ Kazuhiro Kashiwagi, MD, PhD; Akihito Hishikawa, MD; \\ Yasushi Iwao, MD, PhD; Hiroshi Itoh, MD, PhD
}

\begin{abstract}
Background: Cerebrovascular disease is a major cause of mortality and morbidity. Chronic kidney disease (CKD) is prevalent in stroke patients. This study evaluated the correlation between kidney dysfunction and asymptomatic findings on carotid ultrasonography (US) and brain magnetic resonance imaging (MRI) in a Japanese population with health checkups.

Methods and Results: In total, 1,716 subjects aged 40-80 years, who received health checkups from January 1 to December 31 , 2015, were included. Common carotid artery intima-media thickness (CCA-IMT) and carotid plaques by US, and the presence of old non-lacunar infarctions, lacunar infarctions, white matter lesions (WMLs), cerebral microbleeds (CMBs), and atrophy by brain $\mathrm{MRI}$ were evaluated. After adjusting for cardiovascular risk factors, multiple regression analyses revealed that an eGFR ranging from 15 to $44 \mathrm{~mL} / \mathrm{min} / 1.73 \mathrm{~m}^{2}$ was independently associated with CCA plaques and hypoechoic or heterogeneous plaques. Proteinuria was associated with CCA or internal carotid artery plaques, the number of carotid plaques, and the presence of old non-lacunar infarctions and CMBs.
\end{abstract}

Conclusions: Decreased eGFR and proteinuria were independent risk factors for asymptomatic abnormalities on carotid US and brain MRI, which are surrogate markers for cerebrovascular diseases. Evaluation of these abnormalities may be useful for prevention of symptomatic cerebrovascular events in CKD patients.

Key Words: Carotid plaque; Cerebral microbleeds; eGFR; Proteinuria

C hronic kidney disease (CKD) has become a major health problem throughout the world, with a global increase in prevalence to greater than $10 \%$ as reported in $2009,{ }^{1}$ and resulting in almost 1 million deaths worldwide in $2013 .{ }^{2}$ In recent years, CKD has been shown to be a potent cardiovascular and cerebrovascular risk factor. ${ }^{3,4}$ The prevention of these complications is one of the main goals in treating CKD patients.

Cerebrovascular diseases, such as stroke, are major causes of mortality and morbidity. The importance of prevention has been increasingly recognized in aging societies, such as Japan, to extend healthy life expectancy. CKD is prevalent in stroke patients, ${ }^{5}$ and CKD patients show poor functional outcome after ischemic stroke. ${ }^{6}$ Recently, the relationship between kidney dysfunction and neuroimaging findings (e.g., magnetic resonance imaging $(\mathrm{MRI}))^{7}$ has been investigated. Abnormal MRI findings, including lacunar infarcts, white matter lesions (WMLs), cerebral microbleeds (CMBs), and brain atrophy, have not always been associated with apparent symptoms. However, these abnormalities have been correlated with cognitive dysfunction and symptomatic cerebrovascular diseases. ${ }^{5}$ Abnormal findings on carotid ultrasonography (US), including carotid artery intima-media thickness (IMT) and plaques, are also considered surrogate measures of cerebrovascular diseases. Recently associations between kidney dysfunction and the results of carotid US have been investigated, and it was reported that albuminuria and decreased estimated glomerular filtration rate (eGFR) were associated with the presence of carotid plaques. ${ }^{\mathbf{8} 9}$

Many studies have reported that abnormalities in brain MRI or carotid US were observed in patients on hemodialysis (HD) or with endstage renal disease (ESRD); however, the correlation between kidney dysfunction and these abnormalities in the general population remain to be fully examined. Moreover, CKD patients often have complications, such as hypertension, diabetes, and dyslipidemia, which are independent risk factors for cerebrovascular

Received February 8, 2017; revised manuscript received February 26, 2017; accepted March 7, 2017; released online April 14, 2017 Time for primary review: 12 days

Center for Preventive Medicine, Keio University Hospital, Tokyo (K.H., M.T., K.K., Y.I.); Department of Internal Medicine, School of Medicine, Keio University, Tokyo (K.H., T.K., A.H., H.I.), Japan.

Mailing address: Kaori Hayashi, MD, PhD, Department of Internal Medicine, School of Medicine, Keio University, 35 Shinanomachi, Shinjuku-ku, Tokyo 160-8582, Japan. E-mail: kaorihayashi@keio.jp

ISSN-1346-9843 All rights are reserved to the Japanese Circulation Society. For permissions, please e-mail: cj@j-circ.or.jp 
diseases. Therefore, only a small number of studies have evaluated whether kidney dysfunction is an independent risk factor for symptomatic and asymptomatic cerebrovascular diseases.

The aim of this study was to investigate in a general population whether decreased eGFR and proteinuria were independently associated with abnormal findings on carotid US or brain MRI, using a multiple regression analysis adjusted for other risk factors for cardiovascular and cerebrovascular diseases.

\section{Methods}

\section{Study Population}

Individuals aged 40-80 years who had received thorough medical examinations at Keio University Hospital between January 1 and December 31, 2015, were enrolled. We excluded participants without essential data, including age, sex, body mass index (BMI), systolic blood pressure (BP), diastolic BP, serum chemistries, a self-administered questionnaire and carotid artery US. Individuals with ESRD with an eGFR $<15 \mathrm{~mL} / \mathrm{min} / 1.73 \mathrm{~m}^{2}$ were also excluded. In total, data from 1,716 participants (1,175 males, 541 females) were included and analyzed.

\section{Clinical Evaluation and Laboratory Measurements}

All participants completed a self-administered questionnaire that documented medical history, medications, and lifestyle, including smoking habit. Serum markers, including triglyceride (TG), high-density lipoprotein cholesterol (HDL-C), low-density lipoprotein cholesterol (LDL-C), glucose, and hemoglobin Alc (HbA1c), were measured. Blood samples were collected after fasting overnight and immediately analyzed using an automated clinical chemical analyzer. Urinary protein excretion was examined using dipstick testing. BP was measured in the right upper arm after subjects had rested at least $5 \mathrm{~min}$ in a seated position in the hospital, using an automatic device BP-900 with the combination of Korotkoff sounds method and oscillometric technique (TANITA Co., Tokyo, Japan).

\section{Carotid US}

Common carotid artery IMT (CCA-IMT) and the location and characteristics of carotid plaques were assessed by trained examiners using high-resolution US with a 10.0$\mathrm{MHz}$ linear transducer (LOGIQ S8, GE Healthcare, UK). IMT was defined as the distance between the leading edge of the first echogenic line and that of the second echogenic line of the far wall. The thickest point between the carotid bulb and the CCA was measured as the CCA-IMT, as previously described. ${ }^{\mathbf{8} 10}$ CCA-IMT values $>1.0 \mathrm{~mm}$ were considered significant. ${ }^{11}$ A plaque was defined as a focal thickening lesion with an IMT $>1.0 \mathrm{~mm} .{ }^{10}$ The presence and characteristics of carotid plaques were evaluated in the CCA, carotid bulb, internal carotid artery (ICA), and right subclavian artery. The numbers of plaques in these arteries were counted. Plaque intensity was categorized as hyperechoic, isoechoic, hypoechoic, or heterogeneous. ${ }^{12,13}$ These findings were reviewed by experienced radiologists.

\section{MRI}

MRI was performed using a 3.0-T scanner (SIGNA HDxt3.0T, GE Healthcare, USA). A standardized imaging protocol produced axial T2-weighted images, T1-weighted images, fluid attenuated inversion recovery (FLAIR), and
T2*-weighted images by gradient-recalled echo. CMBs were defined as discrete or isolated punctate hypointense lesions, smaller than $10 \mathrm{~mm}$ on T2*-weighted images. ${ }^{\mathbf{1 4}}$ The association of kidney dysfunction with the presence of CMBs was investigated. Brain atrophy was classified as normal or advanced for age, and the association between kidney dysfunction and presence of advanced atrophy for age was examined. WMLs, including periventricular hyperintensity $(\mathrm{PVH})$ and deep and subcortical white matter hyperintensity (DSWMH), were classified into 5 grades $(0-4)$ according to the Shinohara grading: ${ }^{15}$ grade 1 was considered mild, grades 2-3 as moderate, and grade 4 as severe WML. We adopted the higher grade if the PVH and DSWMH grades were different. The association between kidney dysfunction and WML with moderate or severe grade was investigated. These findings were doublechecked by 2 experienced radiologists.

\section{Definitions}

Urinary protein was examined using dipstick testing and categorized into 5 degrees:,, $- \pm 1+, 2+$, and $3+$. Proteinuria was defined as urine protein $\geq 1+$. eGFR $\left(\mathrm{mL} / \mathrm{min} / 1.73 \mathrm{~m}^{2}\right)$ was calculated using the following equation: $=194 \times$ serum creatinine $(\mathrm{mg} / \mathrm{dL})-1.094 \times$ age (years) $-0.287 \times 0.739$ (for women). ${ }^{16}$ Hypertension was defined as systolic BP $\geq 140 \mathrm{mmHg}$ and/or diastolic BP $\geq 90 \mathrm{mmHg}$ or if the patient had indicated use of antihypertensive drugs in the questionnaire. Diabetes was defined in accordance with the guidelines of the American Diabetes Association as a fasting glucose concentration $\geq 126 \mathrm{mg} / \mathrm{dL}$, HbA1c level $\geq 6.5 \%{ }^{17}$ or use of antihyperglycemic drugs in the questionnaire. Dyslipidemia was defined as HDL-C $<40 \mathrm{mg} / \mathrm{dL}$ and/or LDL $\geq 140 \mathrm{mg} / \mathrm{dL}$ and/or TG $\geq 150 \mathrm{mg} / \mathrm{dL}$ or use of a lipid-lowering medication in the questionnaire.

\section{Statistical Analysis}

Multiple logistic or linear regression analyses were used to investigate associations between abnormal findings on carotid US or brain MRI and eGFR category (eGFR $\geq 60$, eGFR $45-59$ or eGFR $15-45 \mathrm{~mL} / \mathrm{min} / 1.73 \mathrm{~m}^{2}$ ) or albuminuria, adjusted by age, sex, BMI, smoking status, hypertension, diabetes, and dyslipidemia. The $45-59 \mathrm{~mL} / \mathrm{min} / 1.73 \mathrm{~m}^{2}$ category of eGFR included CKD stage G3a. The $15-45 \mathrm{~mL} / \mathrm{min} / 1.73 \mathrm{~m}^{2}$ category of eGFR included CKD stages $\mathrm{G} 3 \mathrm{~b}$ and $\mathrm{G} 4$, because the population in each stage was small. For univariable analyses, analysis of variance (ANOVA) followed by Scheffés post-hoc test and Pearson's test were used for continuous and categorical variables, respectively. Continuous variables are expressed as the mean \pm standard deviation (SD). The significance level for all tests in this study was two-sided 5\%. All statistical analyses were performed using JMP version 12 (SAS Institute Inc., Cary, NC, USA).

\section{Results}

\section{Participant Characteristics}

A total of 9,169 individuals had medical checkups between January 1 and December 31, 2015. Individuals aged 40-80 years were included in this study. We excluded individuals without essential data and those with an eGFR $<15 \mathrm{~mL} / \mathrm{min} / 1.73 \mathrm{~m}^{2}$, as described in the Methods section. A total of 1,716 participants (1,175 males, 541 females) aged $61.8 \pm 9.9$ years were eligible for this study. Table 1 shows the general characteristics of the subjects and the 


\begin{tabular}{|c|c|c|c|c|c|}
\hline & \multirow{2}{*}{ Total } & \multicolumn{3}{|c|}{ eGFR (mL/min/1.73 m²) } & \multirow{2}{*}{$P$ value } \\
\hline & & $\geq 60$ & $45-59$ & $15-44$ & \\
\hline$n(\%)$ & $1,716(100)$ & $1,348(79)$ & 330 (19) & $38(2.2)$ & - \\
\hline Age (years) & $61.8 \pm 9.9$ & $60.3 \pm 10.2$ & $67.1 \pm 8.6$ & $71.7 \pm 6.3$ & $<0.0001$ \\
\hline Sex (male) (\%) & $1,175(68)$ & $906(67)$ & $236(72)$ & $33(87)$ & 0.0154 \\
\hline BMI $\left(\mathrm{kg} / \mathrm{m}^{2}\right)$ & $23.4 \pm 3.4$ & $23.4 \pm 3.5$ & $23.7 \pm 3.2$ & $22.6 \pm 2.8$ & 0.1003 \\
\hline Systolic BP (mmHg) & $122.0 \pm 18.4$ & $120.9 \pm 18.1$ & $125.0 \pm 19.3$ & $120.6 \pm 20.6$ & 0.0015 \\
\hline Diastolic BP (mmHg) & $76.8 \pm 10.7$ & $77.0 \pm 10.7$ & $76.8 \pm 11.0$ & $72.3 \pm 10.4$ & 0.0286 \\
\hline $\mathrm{TG}(\mathrm{mg} / \mathrm{dL})$ & $114.9 \pm 81.2$ & $114.1 \pm 83.4$ & $116.1 \pm 71.4$ & $134.0 \pm 79.5$ & 0.3151 \\
\hline HDL-C (mg/dL) & $56.9 \pm 14.8$ & $57.5 \pm 15.0$ & $55.2 \pm 13.6$ & $50.2 \pm 16.1$ & 0.0009 \\
\hline LDL-C (mg/dL) & $117.3 \pm 28.9$ & $117.6 \pm 28.7$ & $117.9 \pm 29.6$ & $100.7 \pm 29.3$ & 0.0016 \\
\hline Glucose (mg/dL) & $108.7 \pm 19.2$ & $108.5 \pm 19.3$ & $108.8 \pm 16.5$ & $114.2 \pm 32.4$ & 0.2010 \\
\hline HbA1c (\%) & $5.80 \pm 0.64$ & $5.78 \pm 0.66$ & $5.85 \pm 0.56$ & $5.97 \pm 0.66$ & 0.0495 \\
\hline eGFR $\left(\mathrm{mL} / \mathrm{min} / 1.73 \mathrm{~m}^{2}\right)$ & $69.9 \pm 9.8$ & $74.6 \pm 10.9$ & $54.2 \pm 4.0$ & $37.8 \pm 5.6$ & $<0.0001$ \\
\hline Proteinuria & $45(2.6)$ & $32(2.4)$ & $7(2.1)$ & $6(16)$ & $<0.0001$ \\
\hline Hypertension & $718(42)$ & $519(38)$ & $168(51)$ & $31(82)$ & $<0.0001$ \\
\hline Dyslipidemia & $783(46)$ & $599(44)$ & $161(49)$ & $23(61)$ & 0.0639 \\
\hline Diabetes & $273(16)$ & $213(16)$ & $51(15)$ & $9(24)$ & 0.4107 \\
\hline Current smoking & $221(13)$ & $193(14)$ & $23(7.0)$ & $5(13)$ & 0.0017 \\
\hline \multicolumn{6}{|c|}{ Findings on carotid ultrasonography } \\
\hline $\mathrm{CCA}-\mathrm{IMT} \geq 1 \mathrm{~mm}$ & $453(26)$ & $324(24)$ & $108(33)$ & $21(55)$ & $<0.0001$ \\
\hline \multicolumn{6}{|l|}{ Location of carotid plaques } \\
\hline Subclavian artery & 329 (19) & $260(19)$ & $63(18)$ & $6(16)$ & 0.8635 \\
\hline $\mathrm{CCA}$ & $174(10)$ & $115(8.5)$ & $44(13)$ & $15(39)$ & $<0.0001$ \\
\hline ICA & $445(26)$ & $316(23)$ & 109 (33) & $20(53)$ & $<0.0001$ \\
\hline Carotid bulb & $1,255(73)$ & $946(70)$ & $275(83)$ & $34(89)$ & $<0.0001$ \\
\hline Hypoechoic or heterogeneous & $120(7.0)$ & $74(5.5)$ & $34(10)$ & $12(32)$ & $<0.0001$ \\
\hline No. of carotid plaques & $1.9 \pm 1.5$ & $1.8 \pm 1.5$ & $2.3 \pm 1.5$ & $3.2 \pm 2.0$ & $<0.0001$ \\
\hline \multicolumn{6}{|l|}{ Findings on brain MRI } \\
\hline Old non-lacunar infarctions & $48(3.5)$ & $30(2.8)$ & $15(5.9)$ & $3(11)$ & 0.0050 \\
\hline Lacunar infarctions & $64(4.7)$ & $44(4.1)$ & $17(6.7)$ & $3(11)$ & 0.0582 \\
\hline White matter lesions & 239 (18) & $184(17)$ & $52(20)$ & $3(11)$ & 0.2891 \\
\hline Cerebral microbleeds & $73(5.4)$ & $54(5.0)$ & $13(5.1)$ & $6(22)$ & 0.0004 \\
\hline Atrophy & $35(2.6)$ & $28(2.6)$ & $6(2.4)$ & $1(3.7)$ & 0.9120 \\
\hline
\end{tabular}

Hypertension, diabetes, dyslipidemia were defined as described in the Methods section. BMI, body mass index; BP, blood pressure; CCA-IMT, common carotid artery intima media thickness; eGFR, estimated glomerular filtration rate; HbA1c, hemoglobin A1c; HDL-C, high-density lipoprotein cholesterol; ICA, internal carotid artery; LDL-C, low-density lipoprotein cholesterol; TG, triglycerides.

\begin{tabular}{|c|c|c|c|c|c|c|}
\hline \multirow{2}{*}{ eGFR (mL/min/1.73 m²) } & \multicolumn{2}{|c|}{$\geq 60$} & \multicolumn{2}{|c|}{$45-59$} & \multicolumn{2}{|c|}{$15-44$} \\
\hline & OR $(95 \% \mathrm{Cl})$ & $P$ value & OR $(95 \% \mathrm{Cl})$ & $P$ value & OR $(95 \% \mathrm{Cl})$ & $P$ value \\
\hline CCA-IMT & 1 & - & $0.85(0.63-1.14)$ & 0.2789 & $1.47(0.72-3.05)$ & 0.2841 \\
\hline Subclavian artery plaque & 1 & - & $1.05(0.76-1.45)$ & 0.7563 & $0.95(0.34-2.22)$ & 0.9072 \\
\hline CCA plaque & 1 & - & $1.06(0.71-1.58)$ & 0.7462 & $2.38(1.10-5.00)^{*}$ & $0.0274^{*}$ \\
\hline ICA plaque & 1 & - & $1.02(0.76-1.36)$ & 0.8755 & $1.21(0.60-2.46)$ & 0.5951 \\
\hline Carotid bulb plaque & 1 & - & $1.13(0.81-1.61)$ & 0.4796 & $0.95(0.35-3.34)$ & 0.9239 \\
\hline Hypoechoic or heterogeneous & 1 & - & $1.33(0.84-2.08)$ & 0.2247 & $2.99(1.31-6.48)^{\star}$ & $0.0100^{*}$ \\
\hline
\end{tabular}

Multivariate logistic regression analyses were performed adjusted by age, sex, BMI, current smoking, hypertension, diabetes, dyslipidemia and proteinuria. *Significant differences. Cl, confidence interval; OR, odds ratio. Other abbreviations as in Table 1.

relationship between these characteristics and eGFR categories. In subjects with a low eGFR, age, systolic BP, $\mathrm{HbAlc}$ and the proportion of male participants, participants with proteinuria, and hypertension were higher and diastolic BP, HDL-C, and LDL-C, and the proportion of current smokers were lower. Carotid US findings showed that the proportions of subjects with CCA-IMT, plaques in CCA, ICA and carotid bulb, hypoechoic or heterogeneous plaques and the number of plaques were significantly higher in the low eGFR category, whereas the 
Table 3. Association Between Proteinuria and Findings on Carotid Ultrasonography Analyzed by Multiple Regression Model

\begin{tabular}{lccccc} 
& \multicolumn{2}{c}{ Proteinuria (-) } & & \multicolumn{2}{c}{ Proteinuria (+) } \\
\cline { 2 - 3 } \cline { 5 - 6 } CCA-IMT & OR (95\% Cl) & P value & OR (95\% Cl) & P value \\
Subclavian artery plaque & 1 & - & & $0.81(0.37-1.69)$ & 0.5839 \\
CCA plaque & 1 & - & & $0.67(0.23-1.59)$ & 0.3892 \\
ICA plaque & 1 & - & & $2.75(1.24-5.84)^{*}$ & $0.0136^{*}$ \\
Carotid sinus plaque & 1 & - & & $2.15(1.07-4.27)^{*}$ & $0.0314^{*}$ \\
Hypoechoic or heterogeneous & 1 & - & & $0.93(0.44-2.02)$ & 0.8455 \\
\end{tabular}

Multivariate logistic regression analyses were performed adjusted by age, sex, BMI, current smoking, hypertension, diabetes, dyslipidemia and eGFR category. *Significant differences. Abbreviations as in Tables 1,2.

\begin{tabular}{|c|c|c|c|c|c|c|}
\hline \multirow{2}{*}{ eGFR (mL/min/1.73 m²) } & \multicolumn{2}{|c|}{$\geq 60$} & \multicolumn{2}{|c|}{$45-59$} & \multicolumn{2}{|c|}{$15-44$} \\
\hline & OR $(95 \% \mathrm{Cl})$ & $P$ value & OR $(95 \% \mathrm{Cl})$ & $P$ value & OR $(95 \% \mathrm{Cl})$ & $P$ value \\
\hline Old non-lacunar infarctions & 1 & - & $1.47(0.73-2.84)$ & 0.2708 & $1.75(0.36-6.21)$ & 0.4512 \\
\hline Lacunar infarctions & 1 & - & $1.06(0.56-1.93)$ & 0.8478 & $1.20(0.26-4.06)$ & 0.7889 \\
\hline White matter lesions & 1 & - & $1.18(0.81-1.68)$ & 0.3844 & $0.56(0.13-1.68)$ & 0.3233 \\
\hline Cerebral microbleeds & 1 & - & $0.67(0.33-1.26)$ & 0.2199 & $1.73(0.55-4.81)$ & 0.3296 \\
\hline Atrophy & 1 & - & $0.88(0.32-2.12)$ & 0.7931 & $1.44(0.08-8.38)$ & 0.7498 \\
\hline
\end{tabular}

*Significant differences. Multivariate logistic regression analyses were performed adjusted by age, sex, BMI, current smoking, hypertension, diabetes, dyslipidemia and proteinuria. MRI, magnetic resonance imaging. Other abbreviations as in Tables 1,2.

\begin{tabular}{|c|c|c|c|c|}
\hline & \multicolumn{2}{|c|}{ Proteinuria (-) } & \multicolumn{2}{|c|}{ Proteinuria (+) } \\
\hline & OR $(95 \% \mathrm{Cl})$ & $P$ value & OR $(95 \% \mathrm{Cl})$ & $P$ value \\
\hline Old non-lacunar infarctions & 1 & - & $3.39(1.04-9.20)^{\star}$ & $0.0430^{*}$ \\
\hline Lacunar infarctions & 1 & - & $2.23(0.61-6.41)$ & 0.2023 \\
\hline White matter lesions & 1 & - & $0.71(0.24-1.70)$ & 0.4631 \\
\hline Cerebral microbleeds & 1 & - & $3.85(1.48-9.09)^{\star}$ & $0.0071^{*}$ \\
\hline
\end{tabular}

*Significant differences. Multivariate logistic regression analyses were performed adjusted by age, sex, BMI, current smoking, hypertension, diabetes, dyslipidemia and eGFR category. No subjects with proteinuria showed advanced atrophy for age. Abbreviations as in Tables 1,2,4.

proportion of subjects with subclavian artery plaque was not significantly different between the groups. The MRI findings showed that the presence of old non-lacunar infarcts and CMBs were significantly higher in the low eGFR group.

\section{Association Between Kidney Dysfunction and Findings of Carotid US}

The association between decreased eGFR or the presence of proteinuria and carotid US findings was investigated using multiple logistic regression analysis adjusted for various factors, including age, sex, BMI, current sm oking status, hypertension, diabetes, and dyslipidemia (Tables 2,3). The analysis demonstrated that eGFR levels of $15-44 \mathrm{~mL} / \mathrm{min} / 1.73 \mathrm{~m}^{2}$ were significantly associated with the presence of plaques in CCA (odds ratio [OR], 2.38; 95\% confidence interval [CI], 1.10-5.00; $\mathrm{P}=0.0274)$ independent of proteinuria, whereas low eGFR levels were not associated with the presence of plaques in the subclavian artery, ICA or carotid bulb. eGFR levels of $15-44 \mathrm{~mL} / \mathrm{min} / 1.73 \mathrm{~m}^{2}$ were also significantly associated with the presence of hypoechoic or heterogeneous plaques (OR, 2.99; 95\% CI, 1.31-6.48; $\mathrm{P}=0.0100)$. Proteinuria was significantly associated with the presence of CCA or ICA plaques (OR, 2.75; 95\% CI, 1.24-5.84; $\mathrm{P}=0.0136,2.15$; $1.07-4.27 ; \mathrm{P}=0.0314$, respectively). In the multiple linear regression analysis, proteinuria correlated with the number of carotid plaques (coeff., $0.21 ; 95 \%$ CI, $-0.01-0.41$; $\mathrm{P}=0.0435)$. eGFR $15-44 \mathrm{~mL} / \mathrm{min} / 1.73 \mathrm{~m}^{2}$ category marginally correlated with the number of carotid plaques (coeff., $0.25 ; 95 \%$ CI, $-0.04-0.55 ; \mathrm{P}=0.0955$ ).

\section{Association Between Kidney Dysfunction and Findings of Brain MRI}

The association between decreased eGFR or proteinuria and brain MRI findings was investigated using a multiple logistic regression model adjusted for factors, including age, sex, BMI, current smoking, hypertension, diabetes, and dyslipidemia (Tables 4,5). Proteinuria was significantly associated with the presence of old non-lacunar infarctions and CMBs after adjusting for the confounding factors listed above (OR 3.39, 95\% CI 1.04-9.20, $\mathrm{P}=0.0430 ; 3.85$, $1.48-9.09, \mathrm{P}=0.0071$, respectively). 


\section{Discussion}

We investigated the association between kidney dysfunction, including reduced eGFR and proteinuria, and asymptomatic abnormalities in the results of carotid US and brain MRI. Using multiple regression analyses adjusted for age, sex, BMI, smoking status, hypertension, diabetes, and dyslipidemia, this study demonstrated the following: (1) eGFR $15-44 \mathrm{~mL} / \mathrm{min} / 1.73 \mathrm{~m}^{2}$ and proteinuria were independently associated with presence of CCA plaques, (2) eGFR $15-44 \mathrm{~mL} / \mathrm{min} / 1.73 \mathrm{~m}^{2}$ was significantly associated with the presence of hypoechoic or heterogeneous plaques, and (3) proteinuria was associated with the number of carotid plaques and the presence of ICA plaques, old non-lacunar infarctions and CMBs.

Carotid US is widely performed to evaluate the status of atherosclerosis in patients with cardiovascular risk factors, including hypertension, diabetes, and dyslipidemia. A number of clinical studies have demonstrated that carotid plaques and IMT are useful surrogate markers for predicting myocardial infarcts and stroke. ${ }^{18-22}$ Recently, CKD was recognized as a risk factor for these events. Previous studies have reported that low eGFR and albuminuria were associated with the presence of carotid plaque. ${ }^{\mathbf{8 9}} \mathrm{We}$ investigated the associations of eGFR and proteinuria not only with the presence of carotid plaques and CCA-IMT, but also with the location, number and characteristics of plaques. Decreased eGFR were significantly associated with the presence of CCA plaques and hypoechoic or heterogeneous plaques, and proteinuria was associated with the number of plaques and the presence of CCA or ICA plaques. It has been demonstrated that hypoechoic or echolucent plaques are associated with a high risk of ischemic cerebrovascular events. ${ }^{\mathbf{1 2} 23}$ Plaque heterogeneity is also suggested to be associated with symptomatic cerebrovascular events. ${ }^{24}$ Although there is to some extent a variety of evaluations among sonographers, examination of plaque characteristics may be useful to predict the risk of symptomatic cerebrovascular events in CKD patients. In this study both decreased eGFR and proteinuria were not associated with CCA-IMT, as previously reported ${ }^{8} \mathrm{CCA}-$ IMT has previously been considered as not representative for atherosclerotic conditions in atherosclerosis-prone lesions, such as the proximal ICAs or carotid bulbs.,25 Our results suggested that evaluation of plaques, especially CCA and ICA plaques, may be useful in patients with proteinuria or with eGFR $<45 \mathrm{~mL} / \mathrm{min} / 1.73 \mathrm{~m}^{2}$ to consider treatment for asymptomatic atherosclerosis.

It was reported that endothelial dysfunction may be related to the association between albuminuria and carotid plaques, because vascular endothelial damage may cause both atherosclerosis and albuminuria. ${ }^{26}$ Decreased eGFR is a risk factor for cardiovascular disease, ${ }^{3}$ although the mechanism of the relationship between low eGFR and atherosclerosis has not been elucidated. Previous reports have suggested various possibilities in CKD, including increased calcium or phosphorous concentrations ${ }^{27}$ or inflammation. ${ }^{28}$

CMBs are indicative of hemosiderin deposits, which can remain in macrophages for years following a microhemorrhage. Growing evidence suggests a correlation between CMBs and increased stroke risk, especially intracerebral hemorrhage. ${ }^{29}$ In the DECIPHER study, Ovbiagele et al ${ }^{30}$ demonstrated that CKD was associated with the presence of CMBs and the number of CMBs in primary intracranial hemorrhage patients when adjusted for confounding factors, including hypertension and diabetes. They defi ned CKD as an eGFR $<60 \mathrm{~mL} / \mathrm{min} / 1.73 \mathrm{~m}^{2}$; therefore, the influence of proteinuria on CMBs was not clear. Recently, CKD has been associated with CMBs in neurologically normal subjects; ${ }^{31}$ however, the association was made using a multiple regression model adjusted only for age and sex, excluding hypertension and diabetes as confounding factors. This study demonstrated the associations of proteinuria with CMBs in asymptomatic subjects in this study, after adjustment for BMI, hypertension, diabetes, dyslipidemia and current smoking status, which are important risk factors in cerebrovascular diseases. The result suggested the importance of proteinuria as an independent risk factor for CMBs. Careful neuroimaging screening may be necessary in patients with proteinuria despite eGFR levels.

Proteinuria was significantly associated with the presence of old non-lacunar infarctions, and neither decreased eGFR nor proteinuria was associated with lacunar infarctions in this study. The relationship between kidney dysfunction and silent brain infarctions in previous studies is controversial. A Rotterdam scan study of a general population in the Netherlands reported that decreased eGFR levels were not significantly associated with asymptomatic lacunar infarcts.32 On the other hand, Kobayashi et al demonstrated in Japanese populations that subjects with CKD or essential hypertension with eGFR levels from 15 to $29 \mathrm{~mL} / \mathrm{min} / 1.73 \mathrm{~m}^{2}$ were significantly associated with silent brain infarcts compared with subjects with an eGFR $>60 \mathrm{~mL} / \mathrm{min} / 1.73 \mathrm{~m}^{2} .{ }^{33}$ Wada et al found that microalbuminuria was associated with lacunar infarction in brain MRI in a cohort of community-based elderly. ${ }^{34}$ Further studies are needed to evaluate the association of low eGFR or proteinuria with various types of infarctions in general population.

Although previous studies have reported that low eGFR levels were associated with WMLs, ${ }^{32,35}$ significant associations were not shown in the present study. Prior studies estimated the volume of WMLs, whereas this study evaluated the presence of WMLs using a severity scale as described in the Methods section. Future studies are necessary to evaluate any associations between WML grade and kidney dysfunction in the Japanese population. Decreased eGFR has been reported to be associated with brain atrophy in a Japanese population with health checkups; ${ }^{36}$ however, this was not the case in the Rotterdam scan study. ${ }^{\mathbf{3 2}}$ In the present study, low eGFR values and proteinuria did not show significant associations with advanced brain atrophy for age when adjusted for other confounding factors related to cardiovascular diseases.

\section{Study Limitations}

First, this study was unable to observe specific cerebrovascular events. Instead, we observed indicators, including carotid US and brain MRI results. Second, the numbers of subjects with an eGFR $45-59 \mathrm{~mL} / \mathrm{min} / 1.73 \mathrm{~m}^{2}$ and $15-44 \mathrm{~mL} / \mathrm{min} / 1.73 \mathrm{~m}^{2}$ were small compared with the number of subjects with an eGFR $>60 \mathrm{~mL} / \mathrm{min} / 1.73 \mathrm{~m}^{2}$, because of the characteristics of the subjects who underwent annual health checkups. Third, single-morning spot urine collections were used instead of timed urine collections, which would have been preferable.

Despite these limitations, this study revealed that decreased eGFR and proteinuria were independent risk 
factors for asymptomatic findings on carotid US and brain MRI, which are surrogate markers for cerebrovascular diseases. Evaluation of carotid US and brain MRI may be useful for prevention of symptomatic cerebrovascular diseases in CKD patients.

\section{Disclosures}

The authors declare no conflicts of interest related to this study.

\section{Acknowledgments}

We express our gratitude to Dr. Suketaka Momoshima, MD and other radiologists and sonographers of the Department of Radiology for their advice on imaging diagnosis.

\section{Ethical Statement and Informed Consent}

General informed consent was obtained because this study was a noninvasive, retrospective study. The records of participants were made anonymous prior to analyses. The ethics committee of the Keio University School of Medicine approved the study protocol (No. 20140329)

\section{Grants}

Grants for Scientific Research (16K19496) from the Ministry of Education, Culture, Sports, Science and Technology (MEXT) of Japan.

\section{References}

1. Szczech LA, Harmon W, Hostetter TH, Klotman PE, Powe NR, Sedor JR, et al. World Kidney Day 2009: Problems and challenges in the emerging epidemic of kidney disease. J Am Soc Nephrol 2009; 20: 453-455.

2. GBD 2013 Mortality and Causes of Death Collaborators. Global, regional, and national age-sex specific all-cause and cause-specific mortality for 240 causes of death, 1990-2013: A systematic analysis for the Global Burden of Disease Study 2013. Lancet 2015; 385: 117-171.

3. National Kidney Foundation. K/DOQI clinical practice guidelines for chronic kidney disease: Evaluation, classification, and stratification. Am J Kidney Dis 2002; 39(Suppl 1): S1-S266.

4. Sarnak MJ, Levey AS, Schoolwerth AC, Coresh J, Culleton B, Hamm LL, et al. Kidney disease as a risk factor for development of cardiovascular disease: A statement from the American Heart Association Councils on Kidney in Cardiovascular Disease, High Blood Pressure Research, Clinical Cardiology, and Epidemiology and Prevention. Circulation 2003; 108: 2154-2169.

5. Toyoda K. Cerebral small vessel disease and chronic kidney disease. J Stroke 2015; 17: 31-37.

6. Kamouchi M. Stroke features and management in patients with chronic kidney disease. Contrib Nephrol 2013; 179: 92-99.

7. Moodalbail DG, Reiser KA, Detre JA, Schultz RT, Herrington JD, Davatzikos C, et al. Systematic review of structural and functional neuroimaging findings in children and adults with CKD. Clin J Am Soc Nephrol 2013; 8: 1429-1448.

8. Choi SW, Kim HY, Lee YH, Ryu SY, Kweon SS, Rhee JA, et al. eGFR is associated with subclinical atherosclerosis independent of albuminuria: The Dong-gu Study. Atherosclerosis 2010; 212: $661-667$.

9. Rajala U, Laakso M, Paivansalo M, Pelkonen O, Ruokonen A, Suramo I, et al. Associations of microalbuminuria and blood pressure with carotid, aortic and femoral atheromatous plaques in elderly Finns. Diabetes Res Clin Pract 2005; 69: 262-271.

10. Kadota A, Miura K, Okamura T, Fujiyoshi A, Ohkubo T, Kadowaki T, et al. Carotid intima-media thickness and plaque in apparently healthy Japanese individuals with an estimated 10-year absolute risk of CAD death according to the Japan Atherosclerosis Society (JAS) guidelines 2012: The Shiga Epidemiological Study of Subclinical Atherosclerosis (SESSA). J Atheroscler Thromb 2013; 20: 755-766.

11. Barnett HJ, Taylor DW, Eliasziw M, Fox AJ, Ferguson GG, Haynes RB, et al. Benefit of carotid endarterectomy in patients with symptomatic moderate or severe stenosis: North American Symptomatic Carotid Endarterectomy Trial Collaborators. $N$ Engl J Med 1998; 339: 1415-1425.

12. Gronholdt ML, Nordestgaard BG, Schroeder TV, Vorstrup S,
Sillesen H. Ultrasonic echolucent carotid plaques predict future strokes. Circulation 2001; 104: 68-73.

13. Waki H, Masuyama T, Mori H, Maeda T, Kitade K, Moriyasu $\mathrm{K}$, et al. Ultrasonic tissue characterization of the atherosclerotic carotid artery: Histological correlates or carotid integrated backscatter. Circ J 2003; 67: 1013-1016.

14. Greenberg SM, Vernooij MW, Cordonnier C, Viswanathan A, Al-Shahi Salman R, Warach S, et al. Cerebral microbleeds: A guide to detection and interpretation. Lancet Neurol 2009; 8: $165-174$.

15. Shinohara Y, Tohgi H, Hirai S, Terashi A, Fukuuchi Y, Yamaguchi T, et al. Effect of the Ca antagonist nilvadipine on stroke occurrence or recurrence and extension of asymptomatic cerebral infarction in hypertensive patients with or without history of stroke (PICA Study). 1: Design and results at enrollment. Cerebrovasc Dis 2007; 24: 202-209.

16. Matsuo S, Imai E, Horio M, Yasuda Y, Tomita K, Nitta K, et al. Revised equations for estimated GFR from serum creatinine in Japan. Am J Kidney Dis 2009; 53: 982-992.

17. American Diabetes Association. Diagnosis and classification of diabetes mellitus. Diabetes Care 2010; 33(Suppl 1): S62-S69.

18. Sakaguchi M, Kitagawa K, Nagai Y, Yamagami H, Kondo K, Matsushita K, et al. Equivalence of plaque score and intimamedia thickness of carotid ultrasonography for predicting severe coronary artery lesion. Ultrasound Med Biol 2003; 29: 367-371.

19. Nagai Y, Metter EJ, Earley CJ, Kemper MK, Becker LC, Lakatta EG, et al. Increased carotid artery intimal-medial thickness in asymptomatic older subjects with exercise-induced myocardial ischemia. Circulation 1998; 98: 1504-1509.

20. O'Leary DH, Polak JF, Kronmal RA, Manolio TA, Burke GL, Wolfson SK Jr. Carotid-artery intima and media thickness as a risk factor for myocardial infarction and stroke in older adults: Cardiovascular Health Study Collaborative Research Group. $N$ Engl J Med 1999; 340: 14-22.

21. Chambless LE, Heiss G, Folsom AR, Rosamond W, Szklo M, Sharrett AR, et al. Association of coronary heart disease incidence with carotid arterial wall thickness and major risk factors: The Atherosclerosis Risk in Communities (ARIC) Study, 1987-1993. Am J Epidemiol 1997; 146: 483-494.

22. Lorenz MW, Markus HS, Bots ML, Rosvall M, Sitzer M. Prediction of clinical cardiovascular events with carotid intimamedia thickness: A systematic review and meta-analysis. Circulation 2007; 115: 459-467.

23. Mathiesen EB, Bonaa KH, Joakimsen O. Echolucent plaques are associated with high risk of ischemic cerebrovascular events in carotid stenosis: The TROMSO study. Circulation 2001; 103: $2171-2175$.

24. AbuRahma AF, Wulu JT Jr, Crotty B. Carotid plaque ultrasonic heterogeneity and severity of stenosis. Stroke 2002; 33: $1772-1775$.

25. Ishizaka N, Ishizaka $\mathrm{Y}$, Takahashi E, Unuma T, Tooda E, Nagai $\mathrm{R}$, et al. Association between insulin resistance and carotid arteriosclerosis in subjects with normal fasting glucose and normal glucose tolerance. Arterioscler Thromb Vasc Biol 2003; 23: 295-301.

26. Stehouwer CD. Endothelial dysfunction in diabetic nephropathy: State of the art and potential significance for non-diabetic renal disease. Nephrol Dial Transplant 2004; 19: 778-781.

27. Block GA, Port FK. Re-evaluation of risks associated with hyperphosphatemia and hyperparathyroidism in dialysis patients: Recommendations for a change in management. Am J Kidney Dis 2000; 35: 1226-1237.

28. Knight EL, Rimm EB, Pai JK, Rexrode KM, Cannuscio CC, Manson JE, et al. Kidney dysfunction, inflammation, and coronary events: A prospective study. J Am Soc Nephrol 2004; 15: $1897-1903$.

29. Charidimou A, Kakar P, Fox Z, Werring DJ. Cerebral microbleeds and recurrent stroke risk: Systematic review and metaanalysis of prospective ischemic stroke and transient ischemic attack cohorts. Stroke 2013; 44: 995-1001.

30. Ovbiagele B, Wing JJ, Menon RS, Burgess RE, Gibbons MC, Sobotka I, et al. Association of chronic kidney disease with cerebral microbleeds in patients with primary intracerebral hemorrhage. Stroke 2013; 44: 2409-2413.

31. Toyoda G, Bokura H, Mitaki S, Onoda K, Oguro H, Nagai A, et al. Association of mild kidney dysfunction with silent brain lesions in neurologically normal subjects. Cerebrovasc Dis Extra 2015; 5: $22-27$.

32. Ikram MA, Vernooij MW, Hofman A, Niessen WJ, van der Lugt A, Breteler MM. Kidney function is related to cerebral small 
vessel disease. Stroke 2008; 39: 55-61.

33. Kobayashi M, Hirawa N, Yatsu K, Kobayashi Y, Yamamoto Y, Saka S, et al. Relationship between silent brain infarction and chronic kidney disease. Nephrol Dial Transplant 2009; 24: 201-207.

34. Wada M, Nagasawa H, Kurita K, Koyama S, Arawaka S, Kawanami T, et al. Microalbuminuria is a risk factor for cerebral small vessel disease in community-based elderly subjects. $J$ Neurol Sci 2007; 255: 27-34.
35. Khatri M, Wright CB, Nickolas TL, Yoshita M, Paik MC, Kranwinkel G, et al. Chronic kidney disease is associated with white matter hyperintensity volume: The Northern Manhattan Study (NOMAS). Stroke 2007; 38: 3121-3126.

36. Yakushiji Y, Nanri Y, Hirotsu T, Nishihara M, Hara M, Nakajima $\mathbf{J}$, et al. Marked cerebral atrophy is correlated with kidney dysfunction in nondisabled adults. Hypertens Res 2010; 33: $1232-1237$ 